\title{
METHODS FOR QUANTIFYING BREAST CANCER-RELATED LYMPHIEDEMA IN PATIENTS UNDERGOING A CONTRALATERAL PROPHYLACTIC MASTECTOMY
}

\author{
S.A. Roberts, C.L. Brunelle, T.C. Gillespie, A.M. Shui, K.M. Daniell, \\ M.W. Lavoie, G.E. Naoum, A.G. Taghian
}

Department of Radiation Oncology (SAR,TCG,KMD,MWL,GEN,AGT) Massachusetts General Hospital, Harvard Medical School, Boston MA; Department of Physical and Occupational Therapy (CLB) and Biostatistics Center (AMS), Massachusetts General Hospital, Harvard Medical School, Boston, Massachusetts, USA

\section{ABSTRACT}

Patients treated for breast cancer are at risk of developing breast cancer-related lymphedema (BCRL). A significant proportion of patients treated for breast cancer are opting to undergo a contralateral prophylactic mastectomy (CPM). Currently, it remains unclear as to whether the relative volume change $(R V C)$ equation may be used as an alternative to the weight adjusted change (WAC) equation to quantify BCRL in patients who undergo CPM. In order to simplify BCRL screening, our cohort of patients who underwent a CPM $(n=310)$ was matched by BMI to a subset of patients who underwent unilateral breast surgery $(n=310)$. Arm volume measurements were $o b$ tained via an optoelectronic perometer preoperatively, postoperatively, and in the follow-up setting every 6-12 months. The correlation of ipsilateral $R V C$ and $W A C$ values for those who underwent bilateral surgery was calculated $(r=0.60)$. Contralateral WAC values for patients in both cohorts were compared, and there was no significant difference between the two distributions in variance $(p=0.446)$. The $R V C$ equation shows potential to be used to quantify ipsilateral postoperative arm volume changes for patients who undergo a CPM.
However, a larger trial in which $R V C$ and $W A C$ values are prospectively assessed is needed.

Keywords: breast cancer, breast cancer-related lymphedema, contralateral prophylactic mastectomy, screening

Roughly one in five women diagnosed with breast cancer will develop breast cancerrelated lymphedema (BCRL) (1-3). BCRL is characterized by protein-rich fluid accumulating in interstitial space, resulting in regional swelling (3). Established risk factors for BCRL include axillary lymph node dissection (ALND) (4-9), regional lymph node radiation (RLNR) (9-12), and a body mass index (BMI) $\geq 30$ $\mathrm{kg} / \mathrm{m}^{2}$ at time of breast cancer diagnosis (1315). BCRL cannot be cured, and patients are at risk for a lifetime (6). Because of this, at Massachusetts General Hospital (MGH), patients undergoing treatment for breast cancer are prospectively screened for BCRL. To properly screen for and diagnose BCRL, we consider objective arm volume measurements in tandem with patient-reported outcome measures and clinical examinations. To obtain arm volume measurements, we utilize an optoelectronic Perometer. The Perometer uses in- 
frared light beams to measure arm volume, which has been demonstrated to be a reliable and valid way to assess limb volume changes that may be attributed to lymphedema $(16,17)$. Patients undergo bilateral arm measurements preoperatively, postoperatively, and every 612 months in accord with their oncology follow-up visits.

Because the volume of one's arms may change with exercise, behavior, hydration, and weight gain or loss, accurate assessment of BCRL depends on comparison to volume of the contralateral arm as well as pre-operative measurements. Therefore, in 2010, our team developed the Relative Volume Change (RVC) equation to quantify arm volume changes in patients undergoing unilateral breast surgery (16). This equation assesses changes in the ipsilateral arm from pre-operative baseline in relation to changes in the contralateral arm from pre-operative baseline. In short, the RVC $=\left[\left(A_{2} U_{1} /\left(U_{2} A_{1}\right)-1\right]\right.$, where $A_{1}$ and $A_{2}$ are the ipsilateral arm volumes at pre- and postoperative visits, respectively, and $U_{1}$ and $U_{2}$ are the contralateral arm volumes at pre- and postoperative visits, respectively. However, because the RVC relies on the volume of the contralateral arm as a control, the equation was initially created only for patients undergoing unilateral breast surgery. We therefore developed a second equation, the Weight Adjusted Change (WAC) equation, in 2013, in an effort to quantify arm volume changes in patients undergoing bilateral breast surgery (18). The WAC equation uses a patient's overall weight as a control, rather than the volume of the contralateral arm. The WAC $=\left[\left(\mathrm{A}_{2} \mathrm{~W}_{1} /\left(\mathrm{W}_{2} \mathrm{~A}_{1}\right)-1\right]\right.$, where $A_{1}$ and $A_{2}$ are the ipsilateral arm volumes at pre- and postoperative visits, respectively, and $W_{1}$ and $W_{2}$ are the weights at preand postoperative visits, respectively.

Therefore, at MGH, two separate equations (the RVC and the WAC) are currently used to quantify patients' arm volume changes throughout and beyond treatment for breast cancer. For patients who undergo unilateral surgery, the RVC equation is a more accurate tool for assessing arm volume changes than the WAC equation because one's contralateral arm is a better control than one's overall weight. For patients who undergo bilateral breast surgery, theoretically, neither arm can act as a control, making it difficult to accurately assess swelling of the ipsilateral arm. However, this cohort of patients can be subdivided into two groups: those who are diagnosed with bilateral breast cancer, and those who are diagnosed with unilateral breast cancer but opt to undergo a CPM. For patients who are diagnosed with bilateral breast cancer, both arms are at risk of developing BCRL given bilateral nodal surgery +/- RLNR. Therefore, the WAC equation is the most appropriate method to screen this cohort for BCRL as there is no "contralateral arm" to act as a control. However, for the subset of patients who are diagnosed with unilateral breast cancer but opt to undergo a CPM, it is unclear as to whether the RVC may be used to assess ipsilateral arm volume changes. If the RVC can accurately quantify arm volume changes in patients undergoing CPM, the screening process for BCRL would be simplified. The majority of patients could be screened with the RVC equation, reserving the WAC equation for the small percentage of patients diagnosed with bilateral breast cancer.

Women diagnosed with unilateral breast cancer are increasingly opting for a CPM (CPM), even though the incidence of contralateral breast cancer has remained constant over time (19-21). Patients generally opt for a CPM as it greatly reduces the risk of developing contralateral breast cancer (22-24). Despite the growing size of this unique patient population, health care providers still lack a means to confidently screen them for BCRL. On the one hand, the contralateral arm does undergo a mastectomy. However, if the mastectomy does not cause swelling of the contralateral side, that arm may be able to act as a pseudo-control (25). In this case, the RVC would likely be a better tool for these patients. Therefore, the purpose of this study is to determine whether the RVC equation may be used to screen patients who undergo a CPM for BCRL.

MATERIALS AND METHODS

Patient Population 


\begin{tabular}{|l|r|}
\hline \multicolumn{2}{|c|}{ TABLE 1 } \\
Demographics and Treatment-Related \\
Characteristics of the Entire Cohort \\
\hline \multicolumn{2}{|c|}{ Number } \\
& (Range or Percent) \\
\hline Age* & $51.5(24.8,85.8)$ \\
\hline BMI* & $24.5(17.6,47.1)$ \\
\hline Stage & \\
0 & $98(15.8 \%)$ \\
1 & $288(46.5 \%)$ \\
2 & $153(24.7 \%)$ \\
3 & $79(12.7 \%)$ \\
4 & $2(0.3 \%)$ \\
\hline Mastectomy & $370(60.0 \%)$ \\
\hline ALND & $191(30.8 \%)$ \\
\hline Total LN sampled* & $2(0,34)$ \\
\hline RLNR & $189(30.5 \%)$ \\
\hline Chemotherapy & $116(18.7 \%)$ \\
Neoadjuvant & $250(40.3 \%)$ \\
\hline Adjuvant & $27.3(0.9,127.6)$ \\
\hline Time of measurement & \\
(months after surgery)* & \\
\hline & \\
\hline
\end{tabular}

From 2005 to 2017, patients undergoing treatment for primary breast cancer were screened for breast cancer-related lymphedema. All women were recruited at the time of initial presurgical consultation in our multidisciplinary breast oncology clinic. Each patient had a preoperative bilateral arm measurement and at least one postoperative bilateral arm measurement. Patients were excluded from analysis if they were diagnosed with or later developed bilateral breast cancer. Of the overall screened population, 340 patients underwent a CPM. We then matched this cohort of 340 patients to our cohort of patients who underwent unilateral breast surgery by preoperative BMI and time of measurement (months after surgery), resulting in two cohorts of $\mathbf{3 1 0}$ patients each. Thirty patients in the CPM cohort could not be matched to anyone in the unilateral cohort, resulting in their exclusion. Demographic information on the entire (combined) cohort is described in Table 1. All human subject work has been approved by the IRB [\#2008P00054 by the Partners Human Research Committee (Institutional Review
Board)] and oral informed consent for BCRL screening for participation in the research study was obtained at preoperative visit.

\section{Statistical Analyses}

Chi square and Wilcoxon rank sum tests were used to compare categorical and continuous demographic and treatment-related risk factors between the cohort of patients who underwent unilateral surgery and the cohort of patients who underwent bilateral surgery with a CPM. An RVC and a WAC for the ipsilateral arm was calculated at one follow-up visit for each of the 310 patients who underwent a CPM. These values were plotted against one another, and the coefficient of determination (R2) as well as the correlation coefficient (r) were calculated.

Next, a WAC for the contralateral arm was calculated for both the bilateral and unilateral cohorts. To determine if the volume of the arm on the side of the CPM fluctuates to a greater extent than the contralateral arm of patients who underwent unilateral surgery, the distribution of contralateral WAC values of the bilateral cohort was compared to the distribution of those of the unilateral cohort using Levene's test of equal variances. All statistical analyses were performed using RStudio version 1.2.1335.

\section{RESULTS}

\section{Demographics}

Because the two cohorts were matched based on BMI and time of measurement, they did not differ in terms of these variables; the cohorts had a median BMI of 24.5 and 24.4 $\mathrm{kg} / \mathrm{m}^{2}$ (unilateral and CPM groups, respectively) $(\mathrm{p}=0.625)$ and a median measurement follow-up of 27.0 months after surgery $(\mathrm{p}=\mathbf{0 . 4 6 9})$ However, the two groups did differ in terms of other variables. In comparison to patients who underwent unilateral breast surgery, those who received a CPM were significantly younger (46.3 vs. 57.6, $\mathrm{p}<0.001$ ) and underwent much more aggressive treatment for the affected side; a higher percentage of patients under- 


\begin{tabular}{|c|c|c|c|}
\hline \multicolumn{4}{|c|}{$\begin{array}{l}\text { TABLE } 2 \\
\text { Demographies and Treatment-Related Characteristies of the Two Cohorts }\end{array}$} \\
\hline Factor & $\begin{array}{l}\text { Patients who underwent } \\
\text { unilateral surgery } \\
(n=310)\end{array}$ & $\begin{array}{l}\text { Patients who underwent } \\
\text { contralateral prophylactic } \\
\text { mastectomy }(n=310)\end{array}$ & $p$-value \\
\hline Age* $^{*}$ & $57.7(32.8,85.8)$ & $46.3(24.8,73.6)$ & $<0.001$ \\
\hline BMI* & $24.5(17.6,47.1)$ & $24.4(17.6,46.1)$ & 0.625 \\
\hline $\begin{array}{c}\text { Stage } \\
0 \\
1 \\
2 \\
3 \\
4\end{array}$ & $\begin{array}{c}53(17.1 \%) \\
170(54.8 \%) \\
61(19.7 \%) \\
24(7.7 \%) \\
2(0.6 \%)\end{array}$ & $\begin{array}{c}45(14.5 \%) \\
118(38.1 \%) \\
92(29.7 \%) \\
55(17.7 \%) \\
0\end{array}$ & $<0.001$ \\
\hline $\begin{array}{l}\text { Chemotherapy } \\
\text { Neoadjuvant } \\
\text { Adjuvant }\end{array}$ & $\begin{array}{c}28(9.0 \%) \\
95(30.6 \%)\end{array}$ & $\begin{array}{c}88(28.4 \%) \\
155(50.0 \%)\end{array}$ & $\begin{array}{l}<0.001 \\
<0.001\end{array}$ \\
\hline $\begin{array}{l}\text { Measurement follow-up time } \\
\text { (months after surgery)* }\end{array}$ & $27(0.9,107.1)$ & $27.0(1.0,127.6)$ & 0.469 \\
\hline \multicolumn{4}{|c|}{ For the unaffected side } \\
\hline Reconstruction & N/A & $297(95.8 \%)$ & N/A \\
\hline SLNB & N/A & $187(60.5 \%)$ & N/A \\
\hline Total LN sampled* & N/A & $1(0,5)$ & N/A \\
\hline
\end{tabular}

-Abbreviations: ALND, axillary lymph node dissection; SLNB, sentinel lymph node biopsy; LN, lymph nodes; $R L N R$, regional lymph node irradiation.

-p-values calculated were calculated via chi-square tests for categorical variables and via Wilcoxon signed-rank tests for continuous variables.

*Median value shown.

went ALND (40.6\% vs. $21.0 \%$ ) as well as RLNR (42.3\% vs. $18.7 \%$ ). Similarly, patients who underwent a CPM were more likely to undergo both neoadjuvant ( $28.4 \%$ vs. $9.0 \%)$ and adjuvant chemotherapy (50.0\% vs. $30.6 \%)$. These differences can be explained by the fact that women in the CPM cohort were of overall higher stage $(\mathrm{p}<0.001)$ ( Table 2).

In our cohort, the proportion of unilaterally affected patients undergoing a CPM gradually increased each year from 2005-2017, reaching a maximum of $30.3 \%$ in 2014 (Fig. 1). Across all years, the incidence of CPM was $15.8 \%$, which is in line with reported incidences in the literature $(19,26)$.

\section{Ipsilateral RVC vs. Ipsilateral WAC - Bilateral Cohort}

An RVC value and a WAC value were calculated for all patients who underwent a CPM ( $n=310$ patients, 310 measurements, in which each measurement has $1 \mathrm{RVC}$ value and $1 \mathrm{WAC}$ value). RVC and WAC values were plotted against one another and the $\mathrm{R} 2$ value was calculated $(\mathrm{R} 2=0.36)($ Fig. 2$)$. Furthermore, a Pearson correlation coefficient was also calculated $(r=0.602, p<0.001)$, indicating that RVC and WAC values do not correlate strongly enough to be used interchangeably.

\section{Changes in the Unaffected Arm Vs. the Pro-} phylactic Mastectomy Arm

The result of Levene's test demonstrates that there was no significant difference between the two distributions in variance $(p=$ 0.446 ). These distributions are listed in Table 3 and may be visualized in Fig. 3. Therefore, we conclude that throughout treatment, the volume of the arm on the side of the CPM changes in a near-identical way to an arm on a side that did not undergo any surgery. 


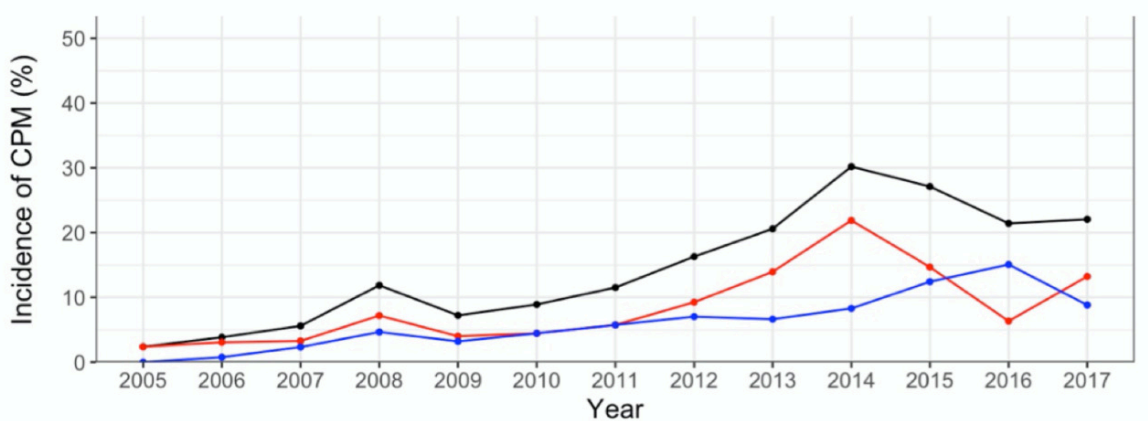

$\rightarrow$ CPM $\rightarrow$ SLNB $\rightarrow$ No nodal surgery

\begin{tabular}{|c|c|}
\hline Year & Incidence \\
\hline 2005 & $2.3 \%$ \\
\hline 2006 & $5.8 \%$ \\
\hline 2007 & $5.8 \%$ \\
\hline 2008 & $11.9 \%$ \\
\hline 2009 & $7.5 \%$ \\
\hline 2010 & $9.3 \%$ \\
\hline 2011 & $13.5 \%$ \\
\hline 2012 & $17.5 \%$ \\
\hline 2013 & $21.7 \%$ \\
\hline 2014 & $30.3 \%$ \\
\hline 2015 & $26.7 \%$ \\
\hline 2016 & $21.9 \%$ \\
\hline 2017 & $21.4 \%$ \\
\hline
\end{tabular}

Fig. 1. Incidence of contralateral prophylactic mastectomies in the cohort of unilaterally affected patients in years 2005 to 2017.

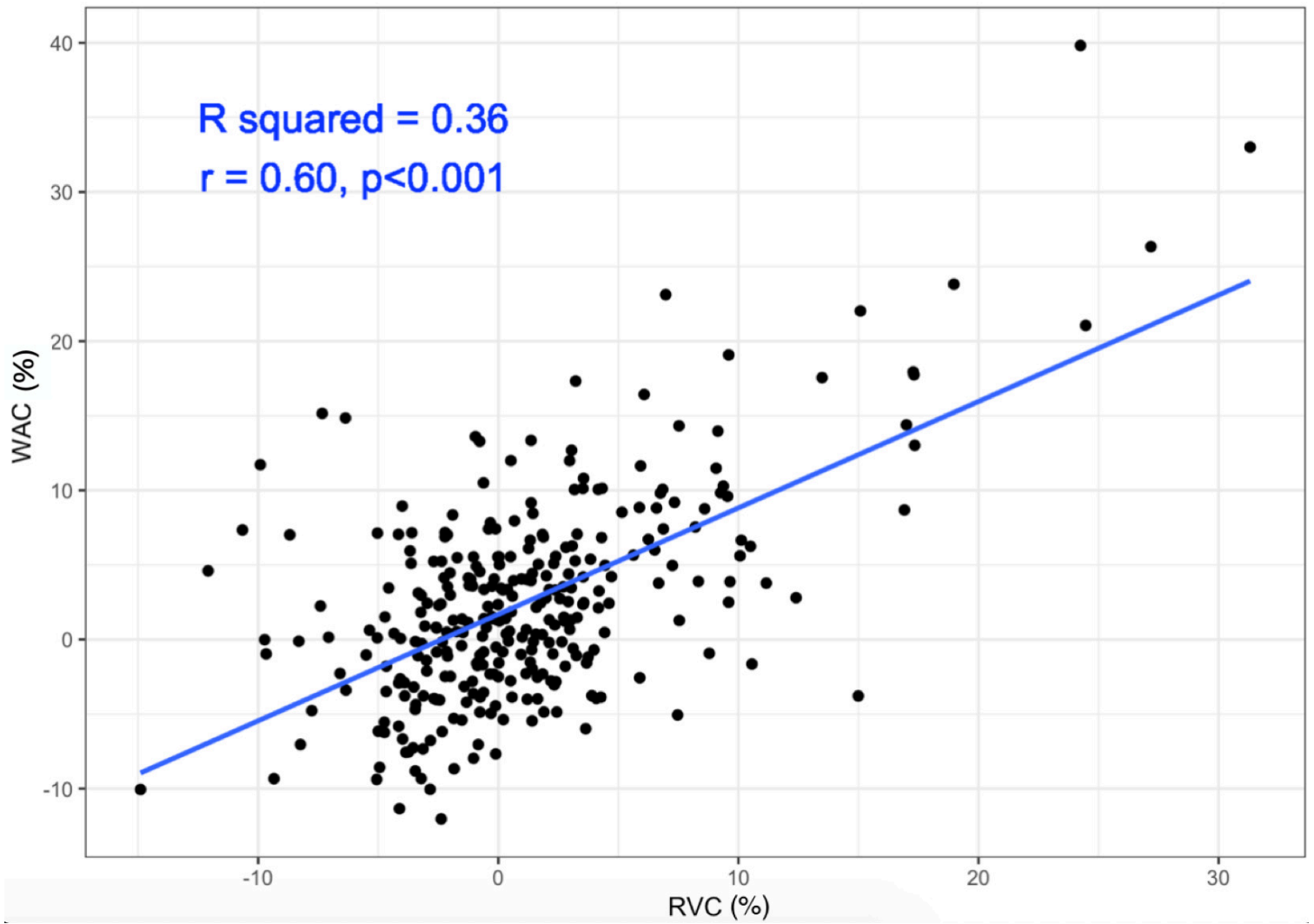

Fig. 2. Correlation of WAC versus $R V C$ values for the affected arm in the cohort who underwent a contralateral prophylactic mastectomy. 


\section{TABLE 3}

Distribution of WACs of the Contralateral Arm

\begin{tabular}{|l|l|l|l|l|l|l|}
\hline & \multicolumn{5}{|c|}{ Percentile } & \multirow{2}{*}{-value } \\
\hline & 0 & 25 & 50 & 75 & 100 & $p$-val \\
\cline { 1 - 5 } Unaffected arm* & $-20.8 \%$ & $-2.9 \%$ & $0.2 \%$ & $3.7 \%$ & $39.4 \%$ & \multirow{2}{*}{0.446} \\
\cline { 1 - 5 } CPM arm** & $-16.3 \%$ & $-2.2 \%$ & $1.0 \%$ & $4.9 \%$ & $24.3 \%$ & \\
\hline
\end{tabular}

*Refers to the contralateral arm of patients who underwent unilateral surgery.

**Refers to the contralateral arm of patients who underwent bilateral surgery.

-Abbreviations: WAC, weight adjusted change; CPM, contralateral prophylactic mastectomy. -p-value was calculated via Levene's test of equal variances.

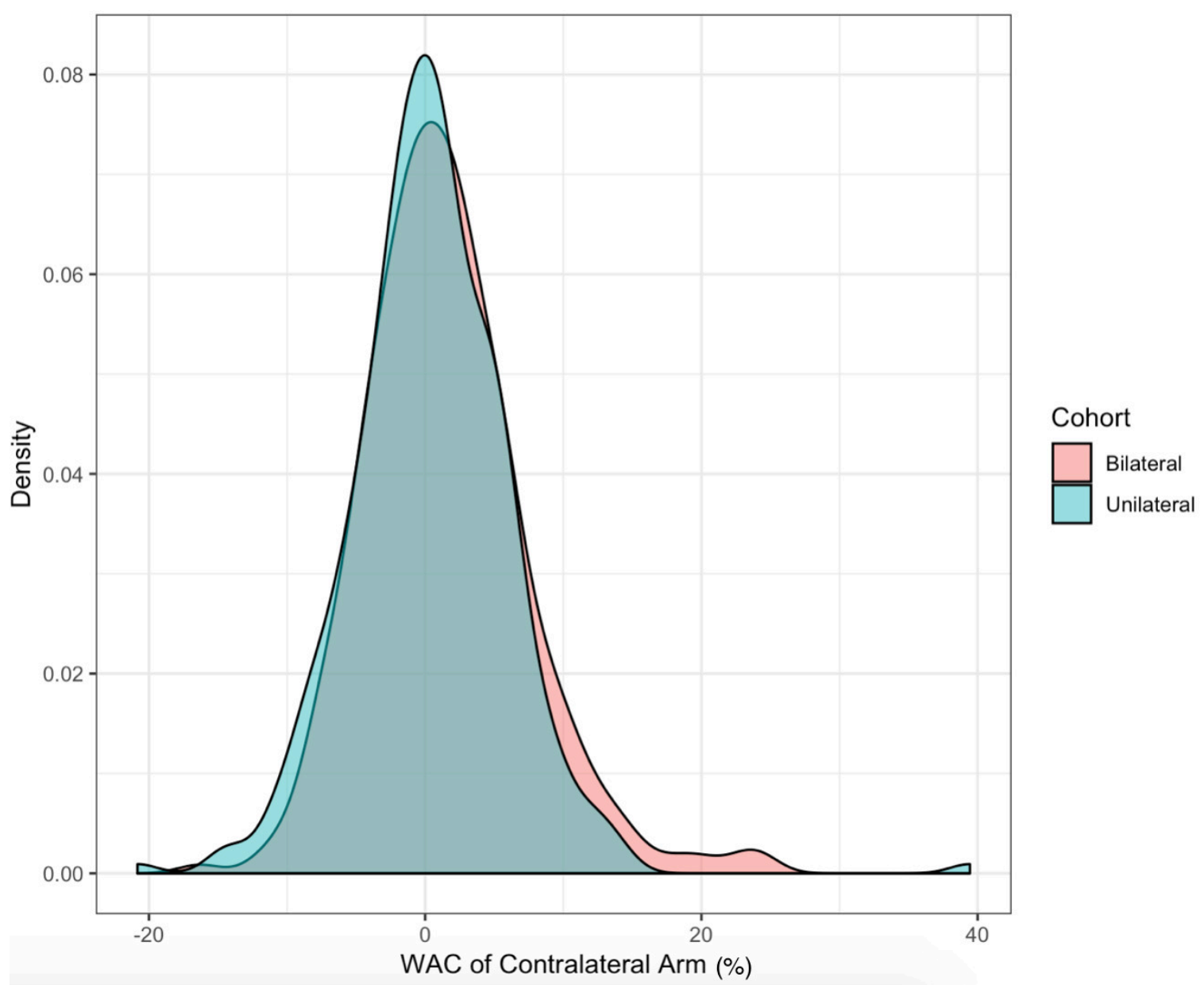

Fig. 3. WAC values of contralateral arms for patients in the bilateral or unilateral cohorts. 


\section{DISCUSSION}

As an increasing number of women opt to undergo a CPM, it is more important than ever to determine how to best screen this cohort of patients for BCRL $(24,26,27)$. While ipsilateral arm volume changes of these patients are traditionally quantified by the WAC equation, we sought to determine if the RVC may be used to simplify screening in this cohort. Ultimately, the basis of answering this question was whether or not the contralateral arm could act as an appropriate control in the RVC equation. The results of our analysis regarding changes in the unaffected arm vs. the prophylactic mastectomy arm indicate that there was no significant difference between the distributions of contralateral WACs in terms of variance in the two cohorts.

The results of these analyses demonstrate that the arm on the side of the prophylactic mastectomy is at low risk of developing BCRL. Therefore, the arm on the side of the CPM may act as a better controlling variable than the patient's weight for calculating the level of swelling of the ipsilateral (affected) arm. In other words, the RVC equation may be an option for screening arm volume changes for the arm on the side of diagnosed cancer for those who opt to undergo a CPM. To reiterate, the RVC equation determines the extent to which the arm on the affected side changes in volume since preoperative baseline, relative to the contralateral arm. Meanwhile, the WAC equation uses one's overall body weight as the controlling variable, instead of the contralateral arm. Of note, the RVC equation should not be used for patients who undergo bilateral surgery for bilateral breast cancer. In this scenario, both breasts are likely to receive extensive oncologic treatment, such as ALND or RNLR, resulting in an elevated risk of BCRL on both sides. For these patients, the WAC equation should be used to monitor volume changes of both arms.

This study is not without limitations. One of the major shortcomings is that the cohorts were not matched by all relevant demographic and treatment related variables. An ideal study would match both cohorts not only by BMI at preoperative baseline and timing of measurements, but also by age at preoperative baseline and percentage of patients undergoing both neoadjuvant and adjuvant chemotherapy. In addition to this, we did not collect information on the lifestyle habits of each patient throughout their treatment for breast cancer. Because lifestyle habits may contribute to arm volume changes in the contralateral arm, this would have been useful information to include in the analyses (28-31). Furthermore, there are other potential risk factors for BCRL such as postoperative seroma or cellulitis that we did not consider.

Altogether, our results suggest that the RVC equation may be used to quantify arm volume changes of the ipsilateral arm for patients who undergo a CPM. However, a larger prospective trial is needed, in which patients are measured at regular intervals and a WAC and RVC are calculated at each visit. At every visit in which an RVC or WAC of $\geq 10 \%$ is discovered, the patient should be seen by a Certified Lymphedema Therapist (CLT) to determine BCRL diagnosis through integration of symptoms, clinical examination, and RVC or WAC. BCRL diagnosis should not be based on arm volume changes alone; symptoms and clinical examination should be integrated with WAC or RVC values to determine whether or not a patient has developed BCRL.

\section{CONFLICT OF INTEREST AND DISCLOSURE}

Taghian is on the Scientific Advisory Board of Puretech Health and a previous Consultant in VisionRT. He has also been loaned equipment from ImpediMed for use in investigator-initiated clinical trials. Cheryl Brunelle is on the Scientific Advisory Board of Puretech Health. However, these associations are unrelated to this manuscript. For the remaining authors, no financial conflicts of interest are declared.

\section{ACKNOWLEDGMENTS}

The project was supported by Award Number R01CA139118 (AG Taghian) and 
Award Number P50CA08393 (AG Taghian) from the National Cancer Institute. The content is solely the responsibility of the authors and does not necessarily represent the official views of the National Cancer Institute or the National Institutes of Health. This program is supported by the Adele McKinnon Research Fund for Breast Cancer-Related Lymphedema (AG Taghian), the Heinz Family Foundation (AG Taghian), and the Olayan-Xefos Family Fund for Breast Cancer Research (AG Taghian).

\section{REFERENCES}

1. American Cancer Society: Breast Cancer Facts \& Figures. 2017-2018.

https://www.cancer.org/research/cancer-factsstatistics/breast-cancer-facts-figures.html.

2. McDuff, SGR, AI Mina, CL Brunelle, et al: Timing of lymphedema after treatment for breast cancer: When are patients most at risk? Int. J. Radiat. Oncol. Biol. Phys. 103 (2019), 62-70. doi:10.1016/j.ijrobp.2018.08.036

3. DiSipio, T, S Rye, B Newman, S Hayes: Incidence of unilateral arm lymphoedema after breast cancer: A systematic review and meta-analysis. Lancet Oncol. 14 (2013), 500515. doi:10.1016/S1470-2045(13)70076-7

4. Fu, MR: Breast cancer-related lymphedema: Symptoms, diagnosis, risk reduction, and management. World J. Clin. Oncol. 5 (2014), 241. doi:10.5306/wjco.v5.i3.241

5. McLaughlin, SA, MJ Wright, KT Morris, et al: Prevalence of lymphedema in women with breast cancer 5 years after sentinel lymph node biopsy or axillary dissection: Patient perceptions and precautionary behaviors. $\mathrm{J}$. Clin. Oncol. 26 (2008), 5220-5226. doi:10.1200/JCO.2008.16.3766

6. Paskett, ED, MJ Naughton, TP McCoy, et al: The epidemiology of arm and hand swelling in premenopausal breast cancer survivors. Cancer Epidemiol. Biomarkers Prev. 16 (2007), 775-782. doi:10.1158/1055-9965.EPI06-0168

7. Petrek, JA, RT Senie, M Peters, P Peterrosen: Lymphedema in a cohort of breast carcinoma survivors 20 years after diagnosis. Cancer 92 (2001), 1368-1377. doi:10.1002/10970142(20010915)92:6<1368::AIDCNCR1459>3.0.CO;2-9

8. Gillespie, TC, HE Sayegh, CL Brunelle, et al:
Breast cancer-related lymphedema: Risk factors, precautionary measures, and treatments. Gland Surg. 7 (2018), 379-403. doi:10.21037/gs.2017.11.04

9. Tsai, RJ, LK Dennis, CF Lynch, et al: The risk of developing arm lymphedema among breast cancer survivors: A meta-analysis of treatment factors. Ann. Surg. Oncol. 16 (2009), 1959-1972. doi:10.1245/s10434-0090452-2

10. Norman, SA, AR Localio, MJ Kallan, et al: Risk factors for lymphedema after breast cancer treatment. Cancer Epidemiol. Biomarkers Prev. 19 (2010), 2734-2746. doi:10.1158/1055-9965.EPI-09-1245

11. Warren, LEG, CL Miller, N Horick, et al: The impact of radiation therapy on the risk of lymphedema after treatment for breast cancer: A prospective cohort study. Int. J. Radiat. Oncol. Biol. Phys. 88 (2014), 565-571. doi:10.1016/j.ijrobp.2013.11.232

12. Kilbreath, SL, KM Refshauge, JM Beith, et al: Risk factors for lymphoedema in women with breast cancer: A large prospective cohort. Breast 28 (2016), 29-36. doi:10.1016/j.breast.2016.04.011

13. Wu, R, $X$ Huang, $X$ Dong, et al: Obese patients have higher risk of breast cancerrelated lymphedema than overweight patients after breast cancer: A meta-analysis. Ann. Transl. Med. 7 (2019), 172-172. doi:10.21037/atm.2019.03.44

14. Jammallo, LS, CL Miller, M Singer, et al: Impact of body mass index and weight fluctuation on lymphedema risk in patients treated for breast cancer. Breast Cancer Res. Treat. 142 (2013), 59-67. doi:10.1007/s10549013-2715-7

15. Ahmed, RL, KH Schmitz, AE Prizment, AR Folsom: Risk factors for lymphedema in breast cancer survivors, the Iowa Women's Health Study. Breast Cancer Res. Treat. 130 (2011), 981-991. doi:10.1007/s10549-0111667-z

16. Ancukiewicz, M, TA Russell, J Otoole, et al: Standardized method for quantification of developing lymphedema in patients treated for breast cancer. Int. J. Radiat. Oncol. Biol. Phys. 79 (2011), 1436-1443. doi:10.1016/j.ijrobp.2010.01.001

17. Tierney, S, M Aslam, K Rennie, P Grace: Infrared optoelectronic volumetry, the ideal way to measure limb volume. Eur. J. Vasc. Endovasc. Surg. 12 (1996), 412-417. doi:10.1016/S1078-5884(96)80005-0 
18. Miller, CL, MC Specht, N Horick, et al: TAGT. A novel, validated method to quantify breast cancer-related lymphedema (BCRL) following bilateral breast surgery. Lymphology 46 (2013), 64-74.

http://www.embase.com/search/results?subact ion=viewrecord \& from=export\&id=L3700512 32\%0Ahttp://resolver.ebscohost.com/openurl? sid=EMBASE\&issn=00247766\&id=doi:\&atitl $\mathrm{e}=\mathrm{A}+$ novel $\% 2 \mathrm{C}+$ validated + method + to + quan tify + Breast + Cancer-

Related+Lymphedema+\%28BCRL\%29+follo wi.

19. Tuttle, TM, S Jarosek, EB Habermann, et al: Increasing rates of contralateral prophylactic mastectomy among patients with ductal carcinoma in situ. J. Clin. Oncol. 27 (2009), 1362-1367. doi:10.1200/JCO.2008.20.1681

20. Schrag, D, KM Kuntz, JE Garber, JC Weeks: Prevention Strategies for Women or BRCA2 Mutations. Cancer. 2000.

21. Nealon, KP, N Sobti, M Gadd, et al: Assessing the additional surgical risk of contralateral prophylactic mastectomy and immediate breast implant reconstruction. Breast Cancer Res. Treat. 179 (2020), 255265. doi:10.1007/s10549-019-05460-0

22. Mcdonnell, BSK, SJ Schaid, JL Myers, et al: Efficacy of contralateral prophylactic mastectomy in women with a personal and family history of breast cancer. J. Clin. Oncol. 19 (2001), 3938-3943.

23. Herrinton, LJ, WE Barlow, O Yu, et al: Efficacy of prophylactic mastectomy in women with unilateral breast cancer: A cancer research network project. J. Clin. Oncol. 23 (2005),4275-4286. doi:10.1200/JCO.2005.10.080

24. Van Sprundel, TC, MK Schmidt, MA Rookus, et al: Risk reduction of contralateral breast cancer and survival after contralateral prophylactic mastectomy in BRCA1 or BRCA2 mutation carriers. Br. J. Cancer 93 (2005), 287-292. doi:10.1038/sj.bjc.6602703

25. Fontein, DB, A Charehbili, JW Nortier, et al: Efficacy of six month neoadjuvant endocrine therapy in postmenopausal, hormone receptor-positive breast cancer patients-a phase II trial. Eur J. Cancer 50 (2014), 21902200. doi:10.1016/j.ejca.2014.05.010
26. Tuttle. TM, EB Habermann, EH Grund, et al: Increasing use of contralateral prophylactic mastectomy for breast cancer patients: A trend toward more aggressive surgical treatment. J. Clin. Oncol. 25 (2007), 52035209. doi:10.1200/JCO.2007.12.3141

27. Peralta, EA, JDI Ellenhorn, LD Wagman, et al: Contralateral prophylactic mastectomy improves the outcome of selected patients undergoing mastectomy for breast cancer. Am. J. Surg. 180 (2000), 439-445. doi:10.1016/S0002-9610(00)00505-5

28. Schwartz, AL: Exercise and weight gain in breast cancer patients receiving chemotherapy. Cancer Pract. 8 (2000), 231237. doi:10.1046/j.1523-5394.2000.85007.x

29. Saquib, N, SW Flatt, L Natarajan, et al: Weight gain and recovery of pre-cancer weight after breast cancer treatments: Evidence from the women's healthy eating and living (WHEL) study. Breast Cancer Res. Treat. 105 (2007), 177-186. doi:10.1007/s10549-006-9442-2

30. Kroenke, CH, WY Chen, B Rosner, MD Holmes: Weight, weight gain, and survival after breast cancer diagnosis. J. Clin. Oncol. 23 (2005), 1370-1378. doi:10.1200/JCO.2005.01.079

31. Schmitz, KH, AB Troxel, A Cheville, et al: Physical activity and lymphedema (the PAL trial): Assessing the safety of progressive strength training in breast cancer survivors. Contemp. Clin. Trials. 30 (2009), 233-245. doi:10.1016/j.cct.2009.01.001

Alphonse G. Taghian, MD, PhD
Professor of Radiation Oncology
Harvard Medical School
Department of Radiation Oncology
Director, Lymphedema Research
Program
Massachusetts General Hospital
100 Blossom Street
Boston, MA 02114
Tel: 617-726-7559
Fax: 617-726-3603
Email: ATAGHIAN@mgh.harvard.edu

